D. Breuker gave a talk on information in transposition tables, based on a paper written with J. Uiterwijk and H.J. van den Herik. After presenting the current practice and options in implementing a transposition table, Breuker explained the results of his experiments to establish the relative importance of the pieces of information stored in the transposition table. One interesting finding was that storing bounds is more important than storing the best move. Since doubling the number of entries of a transposition table is not beneficial beyond a certain point, increasing the information stored per entry seems justified. Breuker experimented with storing the PV of a node, rather than storing only the best move, and discovered that only small improvements are possible.

Finally, $\mathrm{H}$. Iida presented a paper on the gains and risks of opponent model search, a paper co-authored by I. Kotani, J. Uiterwijk and H.J. van den Herik. A more general algorithm than OM search was presented: $\mathrm{OM}^{*}$. Simulation experiments were run in order to determine which error likelihood and strength differences provide $\mathrm{OM}^{*}$ search with gains or risks. The conclusion of the experiments confirms the intuitive hypothesis: $\mathrm{OM}^{*}$ is an appropriate algorithm for playing weaker opponents, but imposes risks when applied against a stronger opponent. It would be very interesting to see how $\mathrm{OM}^{*}$ search performs in a real chess program.

All in all, the conference was very interesting, with many ideas exchanged amongst the conference participants. We are looking forward to the next Advances in Computer Chess conference in 1999.

\title{
THE EXHIBITION GAMES
}

D.M. Breuker and H.J. van den Herik

\author{
MChess Pro 5.0 - IGM David Bronstein \\ Maastricht, ACC8, game 1 \\ 25 minutes per player \\ June 27, 1996, Scandinavian Defence
}

1. e4 d5 2. exd5 Qxd5 3. Nc3 Qd6 4. d4 Nf6 5. Bg5 a6 6. Nf3 Nd7 7. Qd2 b5 8. a4 b4 9. Na2 e6 10. Bf4

Qb6 11. Be2 Bb7 12. 0-0 Be7 13. c3 bxc3 14. bxc3 c5 15. Rb1 Qa7 16. Ne5 0-0 17. Nxd7 Nxd7 18. Qd3

Nf6 19. Rd1 Rd8 20. Be5 Bc6 21. a5 cxd4 22. cxd4 Bb5 23. Qf3 Bxe2 24. Qxe2 Rc8 25. Re1 Nd5 26. Rb1

Rc6 27. Qg4 Bf8 (According to MCHEss Black could win a Pawn with 27. ... f6 28. Bg3 f5 29. Qf3 (29. Qd1? f4) 29. ... Qxd4. After the game IGM Bronstein stated that he did not like to weaken his position with the risk of losing the game.) 28. Rc1 Qd7 29. h3 Rc8 30. Rxc6 Qxc6 31. Qf3 f6 32. Bg3 Qa4 33. Qe2 Rc6 34. Rc1 Rxc1+ 35. Nxc1 Kf7 36. Qxa6 Qd1+ 37. Qf1 Qa4 38. a6 Nb4 39. Nd3 Qxa6 40. Nxb4 Qxf1+ 41. Kxf1 Bxb4 $1 / 2^{-1} / 2$ Bronstein: "A classical Grandmaster draw".

\section{IGM David Bronstein - MCHEss Pro 5.0}

Maastricht, ACC8, game 2

25 minutes per player

\section{June 27, 1996, French Defence}

1. e4 e6 2. d4 d5 3. Nc3 Bb4 4. Ne2 dxe4 (Afterwards IGM Bronstein stated that he played Ne2 "in honour of Lasker, who played this variation a few times in 1935/36.") 5. a3 Bxc3+ (A bad move, according to IGM Bronstein. "Now the black squares at the black king side become weak. For me it is easy: Rook=5, Bishop=4, Knight=3.") 6. Nxc3 Nc6 7. Bb5 Ne7 8. Bg5 f6 9. Be3 0-0 10. Qd2 f5 11. 0-0-0 a6 12. Be2 Rb8 13. f3 exf3 14. gxf3 b5 ("14. gxf3 is a risky move. 14. Bxf3 is much safer.") 15. Rg1 b4 16. axb4 Nxb4 17. Bh6 Ng6 18. Bg5 Qd6 19. h4 c5 20. h5 Qxd4 ("Now 21. hxg6 loses to a cheap trick: 21. ... Nxa2+. At the end of my calculation I always look for cheap tricks.") 21. Qxd4 cxd4 22. Rxd4 Ne5 (Here White plays a beautiful move. The following six moves seem to be forced and afterwards MCHESS is lost.) 23. Be7 Nc6 24. Bxf8 Nxd4 25. Bxg7 Nxe2+ 26. Nxe2 Nxf3 27. Rg3 Kf7 28. Bc3 Nh2 29. Rg7+ Kf8 30. Rxh7 Ng4 31. Nf4 e5 32. Ng6+ Kg8 33. Re7 f4 34. Nxe5 Nxe5 35. Bxe5 Rb5 36. h6 Bf5 37. Rg7+ Kh8 38. Bc3 f3 39. h7 Rc5 40. Rc7+ Rxc3 41. Rxc3 1-0

1 Universiteit Maastricht, Department of Computer Science, P.O. Box 616, 6200 MD Maastricht, The Netherlands. Email: \{breuker, herik\}@cs.unimaas.nl. 